\title{
Системы взвешивания \\ в фармацевтической отрасли Лабораторные весы Cubis II
}

Г. Шварц ${ }^{\top}$

УДК 543.07

Дано подробное описание готового модульного комплекса для взвешивания на базе лабораторных весов Cubis II и пакета приложений QApр компании Sartorius, который соответствует высоким стандартам фармацевтической отрасли по точности, надежности, воспроизводимости и безопасности. Особое внимание уделено вопросам оптимальной передачи, обработки, сохранения и защиты целостности данных в строгом соответствии с нормативными документами. Обсуждаются варианты управления пользователями, электронные подписи, безопасная передача данных, дополнительные средства процедурного контроля и системы долгосрочного хранения результатов измерений.

Ключевые слова: лабораторные весы, фармацевтическая отрасль, пакет приложений QАрр, электронная подпись, целостность данных, функция формирования отчетов, управление пользователями

\section{A detailed description of the finished modular weighing complex based on Cubis II scales and the Sartorius QApp application package is given, which meets the high standards of the pharmaceutical industry in accuracy, reliability, reproducibility and safety. Particular attention is paid to the optimal transmission, processing, storage and protection of data integrity in strict accordance with regulatory documents. Options for user management, electronic signatures, secure data transfer, additional means of procedural control and long-term storage of measurement results are discussed.}

Keywords: laboratory balance, pharmaceutical industry, QApp application package, electronic signature, data integrity, reporting function, user management

\section{Введение}

Серия лабораторных весов Cubis II разработана с учетом возможности индивидуальной конфигурации модулей. Это означает, что из множества аппаратных и программных вариантов пользователь может выбрать и сформировать комбинацию, которая необходима для решения поставленной задачи. На выбор доступны 45 типов модулей для взвешивания,

1 Gempex GmbH, Мангейм, Германия, PCR@Sartorius.com.
7 типов защитных витрин, 2 вида дисплеев и блоков управления, а также 5 пакетов программного обеспечения, включающих более 60 программных приложений.

Соответствие требованиям фармацевтической промышленности обеспечивается благодаря сочетанию современного дисплея МСА с диагональю 7 дюймов $(17,8$ cм) и пакета приложений QApp, специально предназначенного для использования лабораторных систем взвешивания в фармацевтической отрасли (рис. 1). 


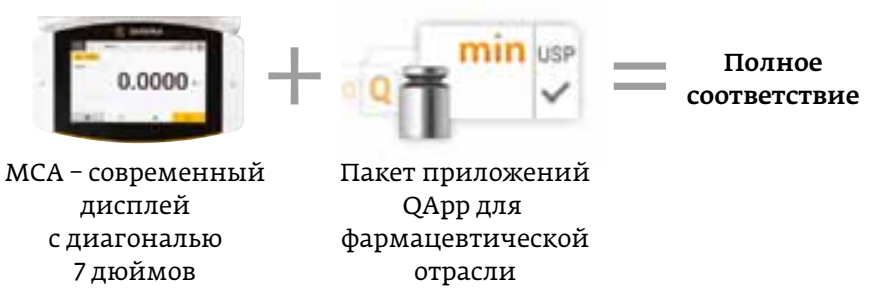

Puc. 1. Готовое решение на базе дисплея MCA и пакета припожений QApp

Конфигурация программного пакета отвечает современным стандартам фармацевтической промышленности и обеспечивает оптимальную передачу, обработку и сохранение целостности данных за счет следующих функций:

- универсальная система управления пользователями для централизованного управления доступом в пределах предприятия с возможностью однократной идентификации;

- полная прослеживаемость операций благодаря наличию системы Audit Trail и функции формирования отчетов;

- легкий переход к электронной форме документооборота за счет использования электронных подписей и нового процесса вывода данных на печать;

- функция автоматического резервного копирования (архивирования) для защиты данных.

Процесс генерации данных в серии весов Cubis II отвечает ключевым принципам обеспечения целостности данных для точного и надежного фиксирования результатов в бумажной и электронной форме (принципы ALCOA (+)). Современная система обработки данных обеспечивает надежное хранение информации различными способами.

Лабораторные весы Cubis II с программным пакетом для фармацевтической отрасли оснащены всеми средствами технического контроля в соответствии

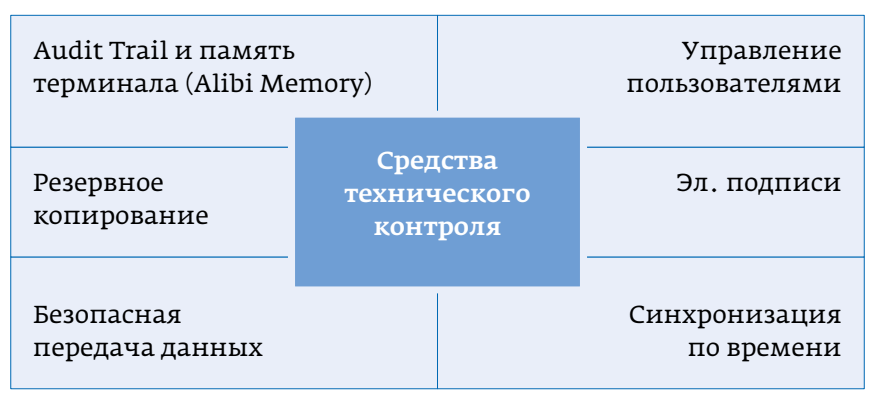

Puc. 2. Средства технического контроля с требованиями части 11 , раздела 21 Свода федеральных нормативных актов CША (21 CFR) и приложения $11 \mathrm{k}$ Требованиям надлежащей производственной практики ЕС. Полное соответствие достигается за счет применения дополнительных средств процедурного контроля и систем долгосрочного хранения данных (рис. 2).

Подробные сведения об этих технических решениях приведены далее.

\section{Управление пользователями}

Доступны два варианта управления доступом пользователей.

Локальное управление пользователями обеспечивается в соответствии с политикой паролей, используемой на предприятии, и предусматривает наличие предварительно заданных неизменяемых ролей (например, "Администратор" или "Оператор"). Кроме того, допускается добавление индивидуальных ролей, а также редактирование прав для каждой роли.

Настройка паролей определяется политикой паролей, принятой в компании (например, путем задания длины, сложности и срока действия пароля). Функция управления пользователями исключает применение ранее использованных паролей. Кроме того, можно настроить автоматический выход из учетной записи после определенного периода бездействия и задать правила в случае достижения максимального числа ошибок при входе в систему.

В системе можно легко задать правила для обеспечения соответствия требованиям части 11 Свода 21 CFR.

Эффективность работы повышается за счет установленных правил создания паролей в активной директории. Система Cubis II может быть внедрена в общую систему предприятия, что обеспечивает возможность однократной идентификации (single sign on, SSO). В этом случае автоматически применяются глобальные правила предоставления паролей. Группы формируются и управляются централизованно, а весь процесс управления внедряется в глобальную рабочую среду компании. Проверка пользователей легко осуществляется силами ИТ-отдела без непосредственного доступа к весам, а добавление или удаление пользователей производится в соответствии с уже установленной процедурой.

\section{Электронные подписи}

В системе Cubis II для подписания итогового отчета о процессе взвешивания предусмотрена электронная подпись (ЭП), основанная на надежном сочетании имени пользователя и пароля. Если политика 
компании допускает равенство ЭП и рукописных подписей, то электронный протокол взвешивания можно включить в общий протокол анализа партии.

\section{Файл Audit Trail}

Audit Trail представляет собой электронный файл данных с метками времени, формируемый на компьютере и защищенный от несанкционированных изменений. Он позволяет восстановить последовательности событий, связанных с созданием, изменением и (или) удалением записей. В целом, сам процесс записи этих данных должен предусматривать защиту от несанкционированного доступа. Если настройки системы отвечают требованиям регулирующих органов, то данные в такой системе должны отображаться в легко читаемом и понятном формате. Программный пакет Pharma фильтрует данные Audit Trail по категориям событий с последующим выводом на экран, что отвечает всем требованиям для регулируемой системы (рис. 3).

Кроме того, весы Cubis II оснащены встроенной памятью терминала; система автоматически сохраняет данные взвешивания в кольцевом буфере, способном вмещать до 150 тыс. массивов данных. Эти записи невозможно удалить или изменить. Однако, их список доступен для просмотра в браузере и может использоваться для дополнительного анализа.

\section{Функция формирования отчетов}

Чтобы протокол взвешивания отвечал нормативным требованиям, в него необходимо добавить дополнительные метаданные: дату и время выборки, версию ПО, идентификатор весов, идентификатор пользователя, номер партии и т. д. Систему можно настроить так, чтобы все необходимые метаданные были включены в надежные записи. Наконец, весь массив данных можно включить в отчет о взвешивании,

\begin{tabular}{|c|c|c|c|}
\hline$<$ Audit Trial & & & $\equiv \downarrow$ \\
\hline $\begin{array}{l}27.02 .2019 \text { г. } \quad 11: 58: 12 \quad \text { (UTC) }\end{array}$ & Администратор & isoCAL & ЮСТИРОВКА КАЛИБРОВКИ \\
\hline 27.02.2019 г. 11:55:47 (UTC) & Администратор & Создано & УПРАВЛЕНИЕ ЗАДАНИЯМИ \\
\hline 27.02.2019 г. 11:54:50 (UTC) & Администратор & Создано & УПРАВЛЕНИЕ QАРP \\
\hline 27.02.2019 г. $10: 20: 41$ (UTC) & Администратор & $\begin{array}{l}\text { Конец } \\
\text { операции }\end{array}$ & $\begin{array}{l}\text { УПРАВЛЕНИЕ } \\
\text { ПОДКЛЮЧЕНИЯМИ }\end{array}$ \\
\hline 27.02 .2019 г. $10: 05: 44$ (UTC) & Администратор & Подпись & КОНТРОЛЬ ДОСТУПА \\
\hline
\end{tabular}

Puc. 3. Фaŭn Audit Trail

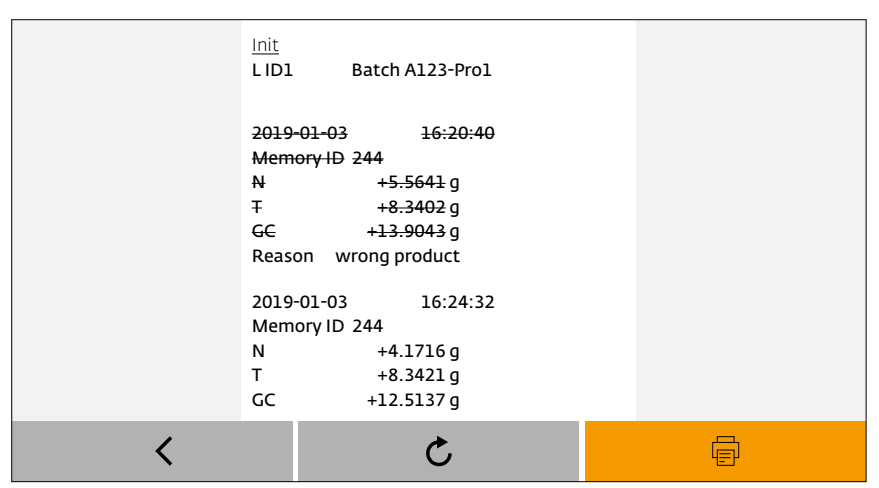

Рис. 4. Протокол взвешивания

составленный в соответствии со стандартами GxP. В процессе печати эти значения включаются в отчет, после чего их можно распечатать или экспортировать в файл PDF с электронной подписью.

В случае ошибки пользователь может пометить массив данных как недействительный, указав пояснительный комментарий перед продолжением работы (рис. 4). Ошибочный массив данных будет отображаться в виде зачеркнутого текста рядом с корректными результатами.

\section{Резервное копирование и архивирование}

Для весов Cubis II возможно автоматическое выполнение регулируемых по времени операций резервного копирования; система способна загружать данные в файл общего доступа или экспортировать их в другие системы. Резервное копирование доступно для Audit Trail, распечатываемых данных, файлов журнала, памяти терминала и конфигурации. Все записи, относящиеся к соблюдению стандартов GxP, надежно сохраняются в системе и могут архивироваться сотрудниками ИТ-отдела в обычном режиме. На случай чрезвычайной ситуации система сохраняет файлы конфигурации для последующего восстановления. Все остальные массивы данных хранятся в файлах формата PDF. Процесс архивирования прост и соответствует нормативным требованиям, обеспечивая возможность чтения всех необходимых дан ${ }^{-}$ ных без использования отдельного ПО или специальных инструментов системы.

\section{Синхронизация по времени}

Надежные записи должны содержать точное время с возможностью проследить его соответствие Всемирному координированному времени (UTC). 
Поэтому весы Cubis II поддерживают автоматическую синхронизацию с помощью протокола сетевого времени (NTP). В большинстве компаний имеются серверы времени, которые предоставляются ИТ-отделом клиента. Вносить изменения в конфигурацию синхронизации по времени могут только уполномоченные лица, действия которых регистрируются системой Audit Trail.

\section{Электронные записи}

Электронная запись должна быть защищена от любых манипуляций. Система Cubis II позволяет выявлять несанкционированные изменения электронных записей благодаря сохранению рассчитанных контрольных сумм MD5 для всех файлов. Алгоритм MD5 широко используется для создания цифровых "отпечатков пальцев" или уникальных идентификаторов для каждого документа. Рассчитанные контрольные суммы сохраняются во всех файлах Audit Trail, а также в отдельном файле MD5. ИТ-системы (например, ЛИС) могут рассчитать контрольную сумму документа и сравнить ее с первоначальной контрольной суммой, полученной от Cubis II. Совпадение этих сумм означает, что с файлами не проводилось никаких манипуляций, то есть результаты взвешивания достоверные.

\section{Интерфейсы}

Аппаратные и программные средства лабораторных весов Cubis II обеспечивают работу практически всех наиболее распространенных настраиваемых интерфейсов. Дополнительные аппаратные средства (например, сканеры штрихкодов, принтеры или накопители) можно подключать к весам с помощью протоколов Ethernet, USB-A, -В и -C, а также через порт последовательной передачи данных по протоколу RS-232. Эти интерфейсы предварительно заданы с целью простого и прямого подключения весов к другим программным системам и устройствам.

Разъемы прибора можно использовать для передачи данных на файловый сервер по различным протоколам (например, Windows File Server (SMB) или Secure File Transfer Protocol (FTPS)). Внешние жесткие диски можно подключать к автономным системам.

\section{Сетевой интерфейс}

Авторизованные пользователи получают доступ к каждым весам Cubis II, подключенным к сети предприятия, через стандартный интернет-браузер

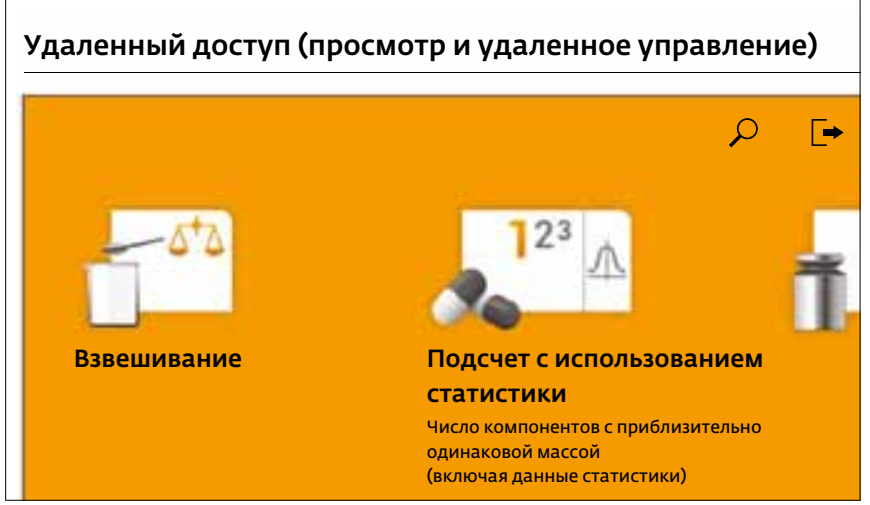

Puс. 5. Удаленный доступ (просмотр и удаленное управление)

с использованием зашифрованного соединения по протоколу HTTPS (рис. 5). Веб-приложение также можно использовать для просмотра данных Audit Trail или памяти терминала.

Этот сетевой интерфейс можно использовать для настройки весов и управления их работой. Функцию удаленного доступа можно отключить, если в ней нет необходимости.

\section{Задачи, профили и разъемы для подключения}

Процедура взвешивания представляет собой сочетание определенных задач и профилей. Пользователь может стандартизировать рабочие процессы путем конфигурации задач для выполнения определен ${ }^{-}$ ной методики. Например, одну задачу можно привязать к двум разным профилям печати. Тогда при выполнении этой задачи происходит экспорт файла в формате PDF в файловый сервер для архивирования данных и на принтер, заданный по умолчанию, для печати протокола взвешивания (рис. 6).

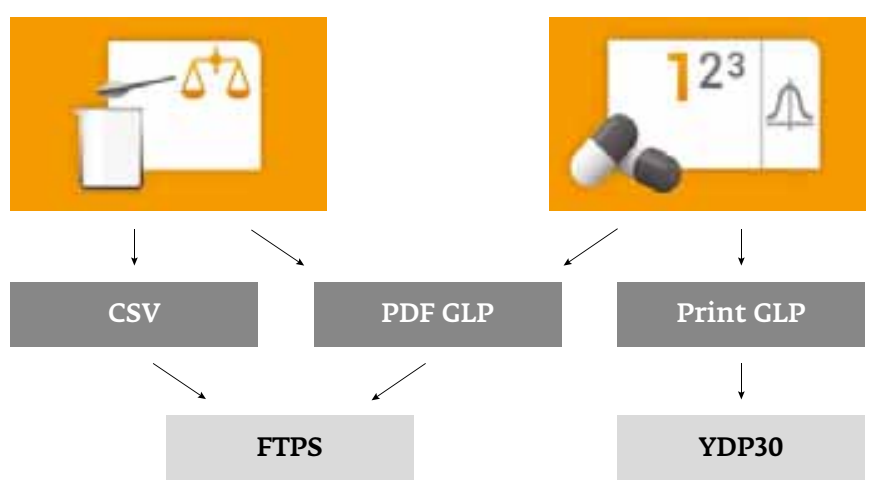

Puc. 6. Возможности использования полученных данных 
Каждый профиль печати связан с определенным разъемом, который может использовать все поддерживаемые Cubis II интерфейсы.

\section{Сохранение протоколов взвешивания}

Для выполнения задач взвешивания можно установить обязательную процедуру внутреннего контроля регулировки весов. Эта настройка позволяет записывать вес только в том случае, если весы правильно отрегулированы. Результаты последней регулировки отображаются в центре статуса весов и записываются в системе Audit Trail. Кроме того, в весах предусмотрена функция проверки выравнивания и автоматическая корректировка при его нарушении.

\section{Заключение}

Конструкция весов серии Cubis II обеспечивает высокую эффективность взвешивания и защиту целостности данных на всех этапах, благодаря приложению QАрр. Кроме того, среди нововведений серии следует отметить отдельные держатели образцов, механизированную систему автоматического выравнивания, встроенный ионизатор для удаления электростатических зарядов и систему управления с функцией распознавания жестов. Центр контроля статуса отображает информацию, предупреждения и сообщения об ошибках, а также условия окружающей среды. Все аппаратные средства разработаны с учетом требований эргономичности и эффективности для получения безошибочных результатов при взвешивании.

Пакет приложений QАрр для фармацевтической отрасли, созданный с учетом рекомендаций GAMP 5 и 21 CFR (Часть 11), обеспечивает работу встроенной системы отслеживания (Audit Trail) и современной системы управления пользователями, а также обработку данных в полном соответствии с нормативными требованиями.

\section{НОВОЕ ИССЛЕДОВАНИЕ МЕТАБОЛИЗМА СОЕДИНЕНИЙ СЕРЫ У БАКТЕРИЙ В ОКЕАНЕ}

Одна из самых активных экосистем планеты - океан, с которым люди редко сталкиваются, а ученые только начинают исследовать. Открытый океан содержит крошечные организмы - фитопланктон, в которых происходит около половины всего фотосинтеза на Земле, помогая генерировать кислород для животных на суше.

Исследование океанографов Вашингтонского университета, опубликованное в журнале Nature Microbiology, посвящено изучению того, как фотосинтетические микробы и океанские бактерии

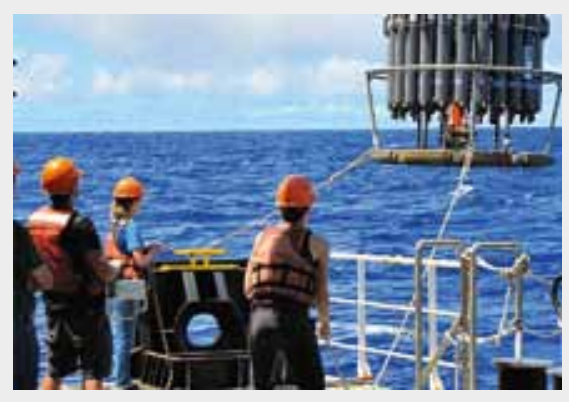

используют серу, богатую морскими питательными веществами.

Сера является пахучим элементом, который придает пляжам их характерный запах. Новое исследование сфокусировано на сульфонатах, в которых атом серы связан с тремя атомами кислорода и молекулой на основе углерода. В океане фитопланктон использует энергию солнца для создания молекул сульфоната. Затем бактерии потребляют сульфонаты для получения питательных веществ и энергии.

Бриндан Дарем, доктор наук в области океанографии, обратился к недавним

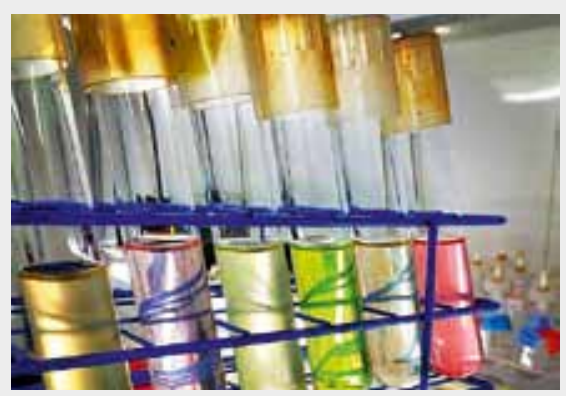

генетическим исследованиям почв, чтобы узнать, какие процессы происходят при переработке сульфонатов в океане. Вначале исследование было сфокусировано на 36 морских микробах, которые группа исследователей культивировала в лаборатории. Использовали метод, разработанный для проверки способности микроорганизмов производить сульфонаты в лабораторных условиях. Затем изучали микробы в открытом океане, поскольку их не удалось вывести в лаборатории.

Исследование обнаружило "некоторые поразительные сходства между сульфонатными путями в наземных и океанических системах». В почвах растения обычно производят сульфонаты. В океанах большинство сульфонатов также продуцируются фотосинтезирующими организмами, в данном случае одноклеточным фитопланктоном.

По материалам: https://environment.uw.edu 


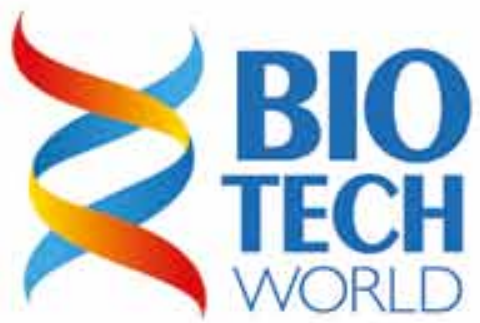

ХVIII МЕЖДУНАРОДНАЯ

СПЕЦИАЛИЗИРОВАННАЯ ВЫСТАВКА

\section{БИОТЕХПРОМ \& АНАЛИТИКА}

\section{РАЗДЕЛЫ ВЫСТАВКИ}

- Аналитика

- БИОФарма

- Функциональные продукты питания

- БИоЛаб

- БИОСофт

- БИОМед

- БИОАгро

- БИОВенчур, БИОФранчайзинг, Стартапы, Кластеры, Технопарки

- Промышленная биотехнология

- Лабораторная мебель БИоЛабДрайв

\section{БИОТЕХНОЛОГИЯ: СОСТОЯНИЕ И \\ ПЕРСПЕКТИВЫ РАЗВИТИЯ}

\section{ОСНОВНЫЕ ПОТОКИ ФОРУМА}

- Фундаментальные вопросы биотехнологии

- Геномная инженерия

- Биотехнология и медицина

- Биофарма

- Биоинформатика

- Сельское хозяйство

- Life science 